responsible for the large Australasian tektite field. One reconciling conclusion is that the meteorite or comet fall (if such there was) leading to the formation of the Australasian tektites produced a number of craters, of which the Darwin Crater is but one. If so, a careful search in the area should reveal them in time.

\section{No role \\ yet for poly (A)}

\section{from our}

Molecular Genetics Correspondent

ONE of the most intriguing questions in the area of messenger RNA production in eukaryotic cells is the role of the sequence of polyadenylic acid (poly (A)) which almost all the messengers seem to carry at their $3^{\prime}$ end. A sequence of about 200 residues of adenylic acid is added, probably one base at a time, to the $3^{\prime}$ end of the apparent mRNA precursor, heterogeneous nuclear RNA (Hn RNA), after or during its synthesis from chromatin. After cleavage of a $3^{\prime}$ terminal sequence containing the poly (A), transport to the cytoplasm is succeeded by association with ribosomes for translation.

One speculation about the possible role of poly (A) is refuted by the results reported by Bard, Efron, Marcus and Perry in the February issue of Cell (1, $103 ; 1974)$. The idea that the poly (A) might be necessary solely for nucleocytoplasmic transport has previously been rendered less likely by observations that it is found in tumour viruses whose expression is confined solely to the cytoplasm and also in mitochondrial messengers where no transport is implicated. One inference which might be drawn from these results is that the poly (A) is implicated in some role in translation, perhaps controlling the stability of mRNA or its capacity to act as template for the ribosomes. Although the sequence of poly (A) on a messenger seems to shorten progressively with time passed in the cytoplasm, studies of mRNA turnover showed that this is not correlated with any increase with time in the probability of mRNA degradation.

The possible role of poly (A) in controlling translational capacity has therefore been examined by Bard et al., using two types of experiment: in the first, they examined the abilities of messengers bearing different lengths of poly (A) to be translated within the mouse $\mathrm{L}$ cell; and in the second they compared the activities as templates in vitro of mRNAs either possessing or stripped of their poly (A) sequences.

Old and new mRNAs can be preferentially labelled in the $\mathrm{L}$ cell cytoplasm by labelling with ${ }^{32} \mathrm{PO}_{4}$ for $20 \mathrm{~h}$ but with ${ }^{3} \mathrm{H}$-adenosine for only $1.75 \mathrm{~h}$. When polysomes are then extracted and analysed, the ${ }^{32} \mathrm{P}$ label identifies older messengers whereas the ${ }^{3} \mathrm{H}$ label is found in newly synthesised messengers. The new messengers possess a length of poly (A) of about 180 residues, but in the old messengers its length is much reduced and may be as small as 50 bases. Bard et al. found that there is no difference in the ratio of new to old $\mathrm{mRNA}$ in various polysomes fractions, implying that both classes of mRNA share the same translational capacity.

An alternative possibility, that overall translational efficiencies are different but that the same ratio of initiation to elongation is maintained, was excluded by experiments utilising temperature shock. This treatment disaggregates polysomes by preventing initiation of protein synthesis; ribosomes therefore run off their current messengers to release free messenger ribonucleoprotein. Lowering the temperature allows the polysomes to reform by utilising the released $\mathrm{mRNP}$. After labelling old $\mathrm{mRNA}$ by incubating cells for $2 \mathrm{~h}$ with ${ }^{3} \mathrm{H}$ uridine followed by a $21 \mathrm{~h}$ chase, and labelling new mRNA by incubation for $1 \mathrm{~h}$ with ${ }^{14} \mathrm{C}$-uridine, Bard et al. found that reassembled polysomes display a ratio of new:old mRNA very close to that of the control. The capacity to initiate protein synthesis of both classes of messenger must therefore be virtually identical.

Because these experiments compare messengers possessing different lengths of poly (A), Bard et al. then turned to in vitro experiments to compare messengers with or completely without poly (A). By using a $3^{\prime}-\mathrm{OH}$ exoribonuclease to degrade the poly (A) sequence, and by then utilising only messengers which had retained their original size (to exclude those suffering other breaks), they isolated a messenger fraction identical to that usually extracted from $\mathrm{L}$ cells, but lacking poly (A). No difference could be found in the translational capacity of the deadenylated mRNA compared with the messengers possessing poly (A), using an in vitro system for synthesising proteins derived from wheat embryo.

Poly (A) therefore seems to lack any role in controlling the activity of messenger RNA in protein synthesis either in vitro or in vivo. The ubiquity of poly (A) in eukaryotic messengers (the only well established fraction lacking it is that coding for the histones) implies that it must have some important function. Previous experiments have excluded control of messenger lifetimes as its function, leaving it with no role apparent at the level of translation. Although preventing the addition of poly (A) halts the production of mRNA from HnRNA, the presence of poly $(A)$ in messengers not derived from the nucleus suggests that it must have some cytoplasmic function. No reconciliation of this paradox is evident at present: poly (A) remains a sequence in search of a function.

\section{Inside the sickled red cell}

\section{from a Correspondent}

CurRent interest in sickle cell anaemia itself and in the behaviour of deoxy sickle cell haemoglobin was the subject of recent comment in these pages (see Nature new Biol., 242, 33; 1973). This interest has been stimulated, partly by increased support in the United States for work on the clinical management of patients with sickle cell anaemia and tests of possible therapy, and partly by new insights into the aggregation of deoxy Hb-S from a variety of techniques, in particular electron microscopy and fibre type X-ray diffraction studies. There is now a considerable literature on the aggregation of deoxy $\mathrm{Hb}-\mathrm{S}$ into an ordered phase, starting with the birefringence studies of Sherman in 1940 , even before the recognition of $\mathrm{Hb}-\mathrm{S}$ as a haemoglobin variant.

One of the latest additions to this literature is an optical study by Hofrichter, Hendricker and Eaton (Proc. natn. Acad. Sci. U.S.A., 70, 3604; 1973) on the orientation of deoxy $\mathrm{Hb}-\mathrm{S}$ molecules within the long straight fibres which have been shown by electron microscopy to make up the ordered phase. The fibre model proposed by Finch and colleagues (ibid., 70,718; 1973) is a microtubule composed of stacked rings of six Hb-S molecules. Since each ring is rotated slightly, relative to the preceding ring, the structure can alternatively be envisaged as six intertwined helical filaments, each having about 48 Hb-S molecules per turn. Neither electron microscopy nor $\mathrm{X}$-ray diffraction has as yet provided definite information on the orientation of the molecules within the fibre, or the role of the mutation sites (Glu $\mathrm{A} 3(6) \beta \rightarrow \mathrm{Val}$ ) in the intermolecular contacts.

Eaton and his colleagues have made polarised absorption measurements on single sickled red cells and single crystals of deoxy Hb-S. The polarisation ratio of sickled cells gives a lower limit for that of an individual fibre, and taken in conjunction with the absorption properties of the haemoglobin molecule restricts the direction of the long molecular axis $(x)$ to within $22^{\circ}$ of the fibre axis. The highly anisotropic absorption ellipsoid of the haemoglobin molecule required for this work was calculated for the Soret band, since optical studies on haem protein single crystals by Faton and colleagues had shown this haem chromophore transition to be nearly perfectly in-plane. The haemoglobin ellipsoid was 